RAPHAËL BARONI

École de français langue étrangère

Université de Lausanne

$\mathrm{CH}-1015$

raphael.baroni[at]unil.ch

\title{
FACE À L'HORREUR DU BATACLAN : RÉCIT INFORMATIF, RÉCIT IMMERSIF ET RÉCIT IMMERGÉ
}

\begin{abstract}
Résumé. - Cet article prolonge le débat ouvert dans la 30 livraison de Questions de communication (2016) concernant la place accordée à la théorie du récit à une époque marquée par un intérêt sans précédent pour des narrations qui s'incarnent dans des formes de plus en plus diverses. Plutôt que d'entrer dans un dialogue avec chaque répondant, j'illustrerai par un exemple concret ce que j'estime être la spécificité d'une démarche qui ne considère pas la théorie du récit comme un simple outil et pour montrer les profits que l'on peut espérer tirer d'un travail de théorisation des formes narratives. Je soutiens qu'il existe deux prototypes opposés de la narrativité, qui forment deux pôles extrêmes entre lesquels se répartissent les représentations narratives suivant que ces dernières se donnent pour tâche principale d'expliquer un événement ou, au contraire, de produire une immersion dans l'expérience racontée. Ces deux prototypes narratifs seront illustrés par deux récits très différents d'un même événement dramatique. J'évoquerai rapidement l'existence d'un troisième prototype : celui du récit immergé dans l'actualité, pour lequel la distinction entre temps du discours et temps diégétique n'a pas lieu d'être. Je reviendrai en conclusion sur les perspectives qui se présentent pour une narratologie de troisième génération.
\end{abstract}

Mołs clés. - narratologie, récit médiatique, intrigue, configuration, immersion, Bataclan 
L'article de Raphaël Baroni « L'empire de la narratologie, ses défis et ses faiblesses 》 $(30,2016)$ a donné lieu à des « Échanges » $(31,2017)$. II revient donc à celui-ci de répondre aux chercheur e s qui ont discuté ses propositions.

\section{De l'utilité de la théorie du récit pour les études narratives}

Cet article prolonge le débat ouvert dans la 30 livraison de Questions de communication (voir Baroni,20 I 6a) concernant la place accordée à la théorie du récit à une époque marquée par un intérêt croissant pour des narrations qui s'incarnent dans des formes de plus en plus diverses, allant des franchises transmédiatiques au storytelling publicitaire ou politique, en passant par tous les genres et les formats que peuvent prendre les récits factuels ou fictionnels qui saturent nos sociétés. Plutôt que d'entrer dans un dialogue avec chaque répondant', j'aimerais illustrer par un exemple concret ce que j'estime être la spécificité d'une démarche qui ne considère pas la théorie du récit comme un simple outil, mais comme un champ de recherche à développer, et pour montrer les profits que l'on peut espérer tirer d'un travail de théorisation des formes narratives. L'analyse comparée de deux traitements journalistiques des attentats qui ont frappé Paris le I 3 novembre 20 I5, qui se focalisent notamment sur l'attaque de la salle de concert du Bataclan, permettra d'éclairer les divergences formelles et fonctionnelles entre deux pôles de narrativité et de rediscuter la thèse ricœurienne concernant l'opération de la « mise en intrigue ».

J'insiste sur le fait que je n'ai aucune critique particulière à adresser à celles et ceux que j'ai nommés maladroitement les « simples usagers » de la boîte à outils narratologique (ibid. : 227). Ainsi que le démontrent Delphine Saurier et OdileVallée (2017), on peut accomplir des analyses très riches de phénomènes intégrant une dimension narrative sans que cela débouche nécessairement sur une théorisation de la narrativité, qui n'est qu'un objectif annexe. II faut d'ailleurs reconnaître qu'au sein des études narratives, la boîte à outils fournie par les narratologues ne représente souvent qu'une part négligeable de l'arsenal conceptuel mobilisé, et que le rendement de ces outils reste dans la plupart des cas modeste, si ce n'est discutable. Ce faible rendement s'explique d'ailleurs non seulement par le caractère limité de ce que l'on peut accomplir avec une perspective narratologique, mais aussi par l'inadéquation de concepts forgés dans le creuset de la théorie littéraire. Les narratologues portent donc une part de responsabilité dans la « faiblesse »

\footnotetext{
Pour les réponses, voir les articles de Jacques Walter et Béatrice Fleury, Jan Baetens, François Jost, Arnaud Schmitt, Marc Marti, Alain Rabatel, Delphine Saurier et Odile Vallée publiés dans la $3 l^{\mathrm{e}}$ livraison de la revue Questions de communication (2017). Je remercie chacun de ces auteurs pour la manière dont ils ont fait avancer le débat par le biais de critiques très constructives. Certains arguments avancés par ces derniers seront rediscutés au fil de cette analyse, notamment dans la dernière partie.
} 
de leur discipline, d'une part, parce qu'ils ont du mal à faire connaître les dernières avancées de leur discipline en dehors des cercles de spécialistes, d'autre part, parce que les récits extra-littéraires ont tardé à être pris au sérieux, ces formes ayant été traitées trop longtemps comme de simples avatars des récits littéraires, au lieu de reconnaître leurs spécificités médiatiques et discursives.

Jan Baetens (2017) souligne à juste titre la dépendance étroite de la théorie envers les objets auxquels elle se confronte : à nouveaux récits, nouvelle théorie. L'élargissement de ce que j'ai désigné un peu pompeusement comme « l'empire de la narratologie » devrait par conséquent engendrer un effort de reconceptualisation qui n'a été jusqu'à présent que très partiellement entrepris. Arnaud Schmitt (2017) rappelle l'importance de l'analyse textuelle, qui a longtemps constitué le principal bénéfice attendu d'un recours à une perspective narratologique, mais Alain Rabatel (2017) souligne aussi la nécessité de décrire beaucoup plus précisément les phénomènes de surface, qui ont été trop souvent négligés, ce qui passe également par une prise en compte plus fine des spécificités sémiotiques et discursives d'objets très divers dans leurs fonctions, leurs formes et leurs fonctionnements. C'est la raison pour laquelle François jost (20 I7) en appelle, et je ne peux que joindre ma voix à la sienne2, à l'émergence d'une véritable narratologie comparée, qui s'attacherait notamment à mieux comprendre les spécificités des incarnations médiatiques des récits, tout en envisageant l'existence de phénomènes qui transcendent ces avatars.

Comme je tenterai de le montrer dans cet article, il me semble en particulier nécessaire de reconnaître l'existence d'une pluralité de prototypes narratifs, de manière à fournir des outils plus maniables et mieux adaptés pour rendre compte des spécificités de récits hétérogènes. Je pense que l'un des torts principaux de la narratologie aura été de chercher à atteindre un prototype universel, ce qui a conduit, dans le meilleur des cas, à négliger le caractère spécifique de certains phénomènes, et dans le pire, à se désintéresser des manifestations qui s'écartent de cette forme idéale ${ }^{3}$.

\section{Deux thèses ricœuriennes discordantes}

Avant d'avancer mes propres arguments, j'aimerais commencer par exposer rapidement la manière dont Paul Ricœur a contribué à ouvrir un nouveau champ de recherche pour l'étude des rapports entre l'expérience temporelle et sa mise en récit, tout en obscurcissant la diversité des formes et des fonctions narratives qui se déploient dans la sphère médiatique. II faut reconnaître que s'il y a bien eu

\footnotetext{
2 Je rejoins cet appel dans Baroni (2017b).

3 J'ai montré ailleurs (Baroni, 20 17b) que le projet d'une narratologie transmédiale a été longtemps contrarié par le dogme genettien voulant que tout récit repose nécessairement sur la production verbale d'un narrateur.
} 
un tournant narrativiste dans la recherche francophone (voir Kreiswirth, 1995), on le doit en grande partie à son œuvre, et notamment à la trilogie Temps et récit publiée entre 1983 et 1985. Avant lui, pour les Anglo-Saxons, c'est surtout le philosophe Hayden White (2017) qui a permis de rapprocher la théorie du récit, traditionnellement confinée à l'étude des fictions littéraires, de la réflexion sur l'écriture des récits factuels, notamment l'historiographie. Cette approche s'inscrivait dans une perspective critique insistant sur l'importance de tenir compte des médiations discursives à travers lesquelles nous rendons compte de la réalité. Ainsi que l'affirme Hayden White, l'écriture n'est jamais transparente : même si elle fait tout pour se faire oublier, elle façonne en partie la réalité dont elle prétend rendre compte. Alors que les travaux de Hayden White, en l'absence de traductions ${ }^{4}$, sont restés largement méconnus du public francophone, Paul Ricœur a joué un rôle de passeur en affirmant à la suite du philosophe américain que la mise en intrigue aurait pour fonction de donner sens et forme à l'expérience temporelle. II en tire la conclusion suivante : « Le temps devient temps humain dans la mesure où il est articulé de manière narrative ; en retour le récit est significatif dans la mesure où il dessine les traits de l'expérience temporelle » (Ricœur, 1983 : 17).

II ne s'agit pas de revenir sur les acquis des approches inspirées de Hayden White et de Paul Ricœur, qui ont eu le double mérite de nous faire prendre conscience de l'omniprésence des formes narratives, qui s'étendent bien au-delà du cercle des fictions littéraires, et de problématiser le rôle des récits dans la manière dont nous nous appréhendons la réalité. Néanmoins, il ne faudrait pas que cette extension conduise à écraser la diversité des récits. On peut soutenir au contraire qu'il existe des différences formelles et fonctionnelles profondes entre la configuration narrative que l'on rencontre dans une fiction littéraire et celle produite par un historien ou un journaliste ${ }^{5}$. Sur ce point, il me semble que la thèse de Paul Ricœur se montre hésitante, voire contradictoire si l'on oppose le premier au troisième tome de Temps et récit'.

Dans le premier tome, la thèse ébauchée dès l'introduction semble se situer dans la droite ligne du rapprochement opéré par Hayden White entre fiction et historiographie. Paul Ricœur (1983 : 12) affirme en effet que « les intrigues que nous inventons » seraient « le moyen privilégié par lequel nous re-configurons notre expérience temporelle confuse, informe et, à la limite, muette ». À ce stade de sa réflexion, Paul Ricœur (ibid. : 126) insiste sur l'importance de généraliser

${ }^{4}$ On doit à Philippe Carrard l'heureuse initiative d'une traduction de quelques textes majeurs de H. White (2017), assortie d'une introduction à ses travaux.

${ }^{5}$ Ce souci de tenir compte des contextes discursif et de la diversité formelle des récits médiatiques se retrouve notamment dans les travaux de Marc Lits (2008) et de Philippe Marion (1997) à qui I'on doit le concept de « médiagénie des récits ». Jean-Michel Adam (201 I) a également insisté sur les variations génériques qui affectent la narrativité.

${ }^{6}$ Pour une discussion critique beaucoup plus détaillée concernant cette contradiction et des conséquences à en tirer pour « poétique comparée des intrigues », je renvoie à Baroni (20 I 0). 
la notion de « mise en intrigue » pour en élargir l'application aux récits factuels, notamment historiques :

\footnotetext{
« Je me propose $[\ldots]$ de dégager cette activité de configuration des contraintes limitatives que le paradigme de la tragédie impose au concept de mise en intrigue chez Aristote. [...] J'espère démontrer par la suite [...] que, sous la condition d'un plus grand degré d'abstraction et avec l'addition de traits temporels appropriés, le modèle aristotélicien ne sera pas radicalement altéré par les amplifications et les corrections que la théorie de l'histoire et la théorie du récit de fiction lui apporteront ».
}

Pour résumer grossièrement, il faudrait donc comprendre cette première thèse de la manière suivante : l'expérience temporelle serait à l'origine confuse, informe et muette, la réponse croisée de la fiction et du récit historique consisterait à transformer cette expérience en l'inscrivant dans une intrigue, autrement dit dans une forme narrative pourvue d'un début, d'un milieu et d'une fin. L'expérience serait ainsi enrichie par la configuration narrative : le temps deviendrait une totalité compréhensible et signifiante grâce à la médiation d'un récit.

Je n'entrerai pas ici dans le détail des problèmes de circularité que pose une telle conception de la narrativité et de l'expérience temporelle, en revanche, j'aimerais souligner le fait que la thèse défendue par Paul Ricœur dans le troisième tome de Temps et récit apparaît sensiblement différente. Au lieu d'insister sur la convergence entre la « mise en intrigue » des récits historiques et des fictions littéraires, Paul Ricœur souligne au contraire l'existence d'une divergence formelle et fonctionnelle profonde entre ces deux formes narratives. II affirme ainsi que la fiction, en proposant des « expériences fictives du temps » forme un « contrepoint avec le monde historique » (Ricœur, 1985 : 229). À l'historien le soin de configurer un récit explicatif qui consiste, entre autres caractéristiques, à se fonder sur des sources pour réinscrire le « temps phénoménologique sur le temps cosmique » (ibid.). À la fiction, au contraire, le rôle d'explorer « les innombrables manières dont l'intentio et la distentio se combattent et s'accordent. En cela, cette littérature est l'instrument irremplaçable d'exploration de la concordance discordante que constitue la cohésion d'une vie » (ibid. : 248-249).

Pour le second Ricœur, l'aporie qui se manifeste à travers la dialectique de la distentio/intentio ou de la concordance/discordance n'est donc plus un problème à résoudre, mais elle constitue la nature même de l'expérience temporelle la plus authentique à laquelle nous reconduirait le récit de fiction. La perspective a donc totalement changé entre le premier et le troisième tome, puisque Paul Ricœur (ibid. : 248) finit par admettre que « la concordance discordante était déjà à la fois le nom d'un phénomène à résoudre et celui de sa solution idéale ». Pour résumer cette nouvelle thèse, on pourrait avancer que face à une expérience temporelle qui n'est que partiellement confuse, puisqu'elle est déjà prise dans des structures qui la rendent signifiante, l'auteur souligne qu'il existe deux types de médiation narrative. La première est d'ordre historiographique : elle consiste à réinscrire l'événement, d'abord vécu comme une expérience subjective, dans un cadre plus large et plus objectif. L'événement devient interprétable, il peut être étayé par 
des sources, on peut en reconstruire l'enchaînement causal ou y reconnaître une configuration déjà connue pour lui attribuer une valeur collective.

Cette opération correspond plus ou moins à la première thèse ricœurienne, mais elle est maintenant restreinte à la description de l'écriture historique. En revanche, la mise en intrigue par la fiction littéraire apparaît pour Paul Ricœur comme une opération très différente : elle vise plutôt à nous rapprocher de l'expérience vécue, à révéler les apories qui se dissimulent derrière les explications plaquées sur les événements. Bref, il n'est plus du tout question de donner sens et forme au vécu, mais plutôt de révéler la nature concordante et discordante de l'expérience imitée par la fiction.

Cette dernière définition rejoint la manière dont je définissais la dynamique de l'intrigue dans La Tension narrative (Baroni, 2007). Dans cette acception étroite, l'intrigue correspond à un dispositif dont la fonction première est d'intriguer le lecteur, et ce faisant, elle met en scène le caractère sous-déterminé de notre expérience temporelle :

« Le suspense met en scène l'incertitude d'un sujet en relation avec son avenir qui est, par nature, sous-déterminé ; la curiosité, pour sa part, est fondée sur l'incertitude d'un objet (qui peut être une chose, un événement, une action ou autrui dans son extériorité) tel qu'il est perçu ou décrit. Le récit serait alors fondamentalement la mise en scène, par le biais de la mise en intrigue, de la sous-détermination du devenir et du monde » (ibid. : | 58).

Le modèle n'est plus celui d'une structure qui configure le passé pour le rendre signifiant, mais plutôt celui d'un arc de tension fondé sur une immersion temporelle dans un événement vécu subjectivement et encore tissé de virtualités et d'incertitudes. Ainsi que l'affirme Richard Pedot (2008: 25), les récits « ne peuvent être réduits au schéma chrono-logique ${ }^{7}$ de l'intrigue sans laisser échapper ce qui en fait la force, la capacité d'intriguer ». Une telle conception rejoint par ailleurs différentes approches linguistiques et narratologiques récentes qui, sous l'influence des modèles cognitivistes, insistent sur le « déplacement déictique » - une opération qui amène le lecteur à se situer imaginairement dans le plan de I'histoire racontée (Duchan, Bruder, Hewitt, 1995) -, sur l'expérientialité qui constitue le cœur de la narrativité mimétique (Fludernik, 1996), sur les virtualités de l'intrigue (Dannenberg, 2008) et sur les calculs probabilistes qui en découlent, qui sont articulés par le lecteur tout au long de sa progression dans l'histoire (Kukkonen, 2014)8. À partir d'une telle perspective, nous verrons comment il est possible de poser les bases d'une stylistique de l'écriture immersive et intrigante,

\footnotetext{
Sur le schéma quinaire, je renvoie aux travaux sur le récit de Jean-Michel Adam (1992: 5I-59). La séquence narrative prototypique telle que définie par la linguistique textuelle n'est pas incompatible avec une approche de l'intrigue comme arc de tension narrative, ainsi que le soulignent les derniers travaux de J.-M. Adam, qui inscrivent l'approche textualiste dans le cadre de l'analyse de discours.

8 Pour une synthèse de ces approches, voir Baroni (2017a) et Patron (2018). Ainsi que le montre P. Carrard (20/2), l'immersion dans une scène narrative et la focalisation sur le destin d'êtres individuels entre en contradiction avec le projet des historiens d'enrichir la compréhension du passé en l'extrayant de la contingence de son déroulement.
} 
et comment on peut l'opposer à une représentation narrative diamétralement opposée, qui cherche au contraire à extraire les événements de la contingence de leur déroulement.

\section{Deux approches de la narrativité : configuration et mise en intrigue}

Je pense que la seconde thèse de Paul Ricœur, malheureusement beaucoup moins populaire que la première, est pourtant la plus convaincante, car elle permet d'éclairer les divergences formelles et fonctionnelles profondes qui existent entre différentes formes narratives. Néanmoins, il me semble que rattacher cette opposition aux genres de la fiction littéraire et du récit historique pose de nombreux problèmes ${ }^{9}$. Premièrement, au sein de ces deux genres, il existe un grand nombre de possibilités narratives. Certains récits historiques peuvent malgré tout jouer sur une immersion dans l'expérience subjective d'un protagoniste des événements, par exemple dans des dramatisations à visée didactique ${ }^{10}$, et il faut ajouter que les codes sur lesquels se fonde l'écriture historique varient d'une culture à une autre ou d'une période à une autre. Par ailleurs, rien n'empêche certaines fictions d'adopter le ton des récits factuels, à l'instar du Marbot de Wolfgang Hildesheimer" '.

II faut ajouter que la tension entre enrichissement explicatif et reconstitution d'une expérience ne concerne pas uniquement les fictions littéraires ou I'historiographie. Marie Vanoost (2016) a montré que les récits journalistiques se répartissent eux aussi entre deux sous-catégories : un journalisme centré sur les faits et leur explication, assez proche de l'opération historiographique classique, et un « journalisme narratif », tiraillé entre le désir d'immerger le lecteur dans une expérience racontée et la nécessité de l'informer en réinscrivant cette représentation dans un cadre plus signifiant ; ces deux sous-catégories pouvant se mêler dans la pratique quotidienne des journalistes (voir aussi Merminod, 20 l 8b). Le récit immersif a donc des affinités évidentes avec la fiction littéraire, mais il n'est pas nécessairement étranger aux genres factuels, dont le spectre inclut aussi l'autobiographie, la biographie romancée, le témoignage ou le reportage.

\footnotetext{
9 II faut cependant noter que pour P. Ricœur récit littéraire et récit historique prennent un sens de plus en plus métaphorique à mesure que progresse sa réflexion, notamment dans Soi-même comme un autre (1990).

10 Je pense notamment à L'Histoire de France en bande dessinée, lancée par Larousse en 1976 et récemment rééditée, ou à la série télévisée documentaire pour la jeunesse Points de repères, diffusée depuis 2016, qui rejoue des moments clés de l'histoire en faisant reposer ces bifurcations sur des décisions subjectives, et en envisageant d'autres alternatives sur le mode de l'uchronie.

" Sir Andrew Marbot est une œuvre de fiction publiée en 198I parWolfgang Hildesheimer. Le livre se présente comme la biographie d'un critique d'art oublié du XIX siècle. Beaucoup de lecteurs ayant lu cette œuvre comme un véritable essai historique et non comme une fiction, elle est parfois considérée comme un canular. Le contrat de lecture proposé par cette œuvre romanesque a été récemment rediscuté par Frank Wagner (2017).
} 
II existerait donc deux prototypes opposés de la narrativitél2 ${ }^{2}$, qui forment deux pôles extrêmes entre lesquels se répartissent les représentations narratives, suivant que ces dernières se donnent pour tâche principale de produire une immersion dans l'expérience racontée ou, au contraire, d'expliquer les événements. On peut décrire la première catégorie comme un récit immersif, de manière à insister sur l'importance du déplacement déictique et de la focalisation sur une expérience simulée ; je pense que l'expression mise en intrigue devrait être réservée à cette catégorie narrative, du moins si l'on souhaite préserver ses connotations en français, l'intrigue étant associée dans la langue naturelle à l'intérêt narratif, au suspense, à la curiosité ou à la surprise. On peut baptiser récit informatif la seconde catégorie, qui désigne un prototype inverse consistant à configurer les événements pour en accroître l'intelligibilité, ce qui rejoint la première thèse défendue par Paul Ricœur ${ }^{13}$ :

I. Récit immersif : L'intrigue est conçue dans le but d'immerger le lecteur dans une expérience simulée et de nouer une tension orientée vers un dénouement éventuel. II s'agit de construire une expérience esthétique fondée sur le suspense, la curiosité ou la surprise ${ }^{14}$, ce qui implique que la compréhension globale des événements est stratégiquement retardée ou définitivement empêchée.

2. Récit informatif : La configuration narrative vise à ordonner le passé d'un point de vue rétrospectif, à établir les faits et à associer les événements à des cadres interprétatifs qui les rendent compréhensibles : causalité, stéréotype, jugement axiologique, exemplarité, etc.

Sur la base de cette opposition, il me semble discutable d'affirmer, comme c'est trop souvent le cas dans le domaine des recherches sur le récit médiatique qui s'inscrivent dans le sillage de Paul Ricœur, que la fonction de la « mise en intrigue » serait de donner sens et forme au réel. II me semble à l'inverse que les formes les plus saillantes de narrativité sont celles qui déploient des trésors d'ingéniosité pour obscurcir, au moins provisoirement, le sens des situations narratives représentées, leur fonction étant plutôt d'intriguer le lecteur et de le (re)plonger au cœur d'une expérience saisie dans le cours de son accomplissement. Je reconnais cependant l'existence d'une autre forme de narrativité, assez rare dans le registre fictionnel, mais très répandue ailleurs, qui vise à configurer les événements pour en accroître l'intelligibilité. L'opposition entre récit informatif et récit immersif rejoint alors celle

\footnotetext{
12 Je discute ces deux prototypes, parfois avec d'autres étiquettes (« récit intrigant 》 ou « récit mimétique » d'un côté, « récit configurant » ou « récit explicatif » de l'autre), dans Baroni (2009; 2017a).

13 Cette typologie se fonde en partie sur la définition gradualiste de la narrativité défendue par Françoise Revaz (2009 : I0 I-137), laquelle souligne à juste titre les différences de nature entre chronique, relation et récit, ce dernier étant la seule forme narrative à reposer sur une véritable mise en intrigue des événements. La spécificité de mon approche tient au fait que les deux prototypes que je dégage ne se distinguent pas par leur degré de complexité, mais par des fonctions discursives opposées.

14 Sur les liens entre les intérêts narratifs et « l'immersion temporelle », voir Ryan (200| : |4| - |48).
} 
introduite par Monika Fludernik (1996:71, ma traduction) entre ce qu'elle appelle le « report » et le « narrative storytelling $»$ :

« II faut établir une distinction entre la forme minimale d'un simple compte rendu des événements (report) et une narration à grande échelle (narrative storytelling). [...] Le compte rendu sert simplement à résumer ou à présenter les faits dans une affaire, à fournir des informations. II est fondé sur une expérience de seconde main ou sur un résumé d'une expérience directe rendue de façon non expérientielle. Il est donc pleinement corrélé avec l'objectivité, la distance et le sens d'une histoire ${ }^{\mid 5} \gg$.

Monika Fludernik fonde sa définition de la narrativité sur la notion d'expérientialité, ce qui explique le caractère plus marginal des comptes rendus dans sa théorie. C'est ce qui la conduit d'ailleurs à exclure les récits historiques du domaine de la narrativité telle qu'elle la définit:

« Je soutiens ici que la narrativité est une fonction des textes narratifs et qu'elle se fonde sur une expérientialité de nature anthropomorphe. [Cette] définition exclut provisoirement l'écriture historique du domaine central de la narrativité prototypique, dans la mesure où l'historiographie consiste en un simple calibrage d'événements qui sont ensuite rapportés comme des faits historiques ${ }^{16} \gg$ (ibid. : 26, ma traduction).

Même si les récits que l'on peut associer à la forme du compte rendu apparaissent moins saillants du point de vue de leur narrativité, il ne faudrait pas en conclure qu'ils seraient moins fondamentaux dans la manière dont nous nous représentons les histoires qui saturent nos existences et les sociétés dans lesquelles nous vivons. II ne faudrait pas davantage négliger la complexité des moyens discursifs visant à accroître l'intelligibilité des événements évoqués dans les récits informatifs, dispositifs qui constituent le contrepoint exact des vecteurs immersifs mobilisés par le prototype opposé.

\section{Trois prototypes narratifs : récit informatif, récit immersif et récit immergé}

Pour illustrer les formes de narrativité dont j'ai esquissé le contour, j'opposerai d'abord deux récits d'un même événement, qui se rattachent l'un comme l'autre au registre du discours journalistique, mais qui se situent aux antipodes si l'on considère leur traitement narratif des événements. Ces deux articles ont été

\footnotetext{
15 «One needs to distinguish between the minimal form of a mere report of events and narrative storytelling on an extended scale. [...] Report is used simply to summarize or present the facts of the case, to provide information. Report is predicated on second-hand experience or on a summary of first-hand experience rendered non-experientially. It therefore fully correlates with objectivity, distance, and the "point" of a story 》.

16 « I here argue that narrativity is a function of narrative texts and centers on experientiality of an anthropomorphic nature. [T] he definition tentatively excludes historical writing from the central realm of prototypical narrativity, namely to the extent that historiography consists in a mere calibration of events which are then reported as historical facts $\gg$.
} 
choisis parce qu'ils renvoient à un événement particulièrement dramatique, qui offre donc potentiellement prise pour la construction d'un récit saillant. Ils offrent par ailleurs, en dépit de cette référence commune, un maximum de contraste dans leur traitement discursif de l'histoire, chacun incarnant idéalement l'un des pôles de la narrativité immersive ou informative. II s'agit de montrer que le traitement médiatique d'un événement singulier peut déboucher sur des productions discursives très variées, et que leur analyse exige également de recourir à des prototypes narratifs différenciés pour en expliquer le fonctionnement. On ne peut donc pas se satisfaire du préjugé qui voudrait que, par le simple fait qu'une histoire serait « racontée », il y aurait nécessairement « mise en intrigue » des événements, ce qui impliquerait à la fois une immersion dans un monde possible et la configuration d'un sens ou d'une explication. II semblerait au contraire que certaines de ces fonctions discursives soient mutuellement exclusives, ou du moins qu'elles reposent sur des rouages narratifs spécifiques.

II faut préciser que l'ancrage discursif des deux récits est très différent : le premier est un article de presse publié dans le quotidien Le Monde le samedi I 4 novembre 20I5, soit le lendemain des attentats du 13-Novembre. Le second est un article du magazine hebdomadaire Les Inrockuptibles, publié cinq jours après les faits, le 18 novembre. Les deux publications n'ont donc pas le même public et ne s'inscrivent pas dans la même temporalité médiatique. Le Monde est un quotidien généraliste qui se rattache à une presse de qualité, ce qui implique que la fonction de divertissement est mise à l'arrière-plan, au profit de la mission d'information et d'explication de l'actualité. Les Inrockuptibles est un magazine hebdomadaire culturel, engagé politiquement, mais dont la fonction de divertissement est plus appuyée. Son rapport à l'actualité est aussi moins direct, ce qui se manifeste, entre autres caractéristiques, par son ouverture à des articles qui privilégient la forme de l'entretien, du reportage ou de ce que Marie Vanoost (voir supra) appelle le « journalisme narratif ». L'orientation culturelle du magazine, qui ménage une place importante à l'actualité musicale, explique aussi que son traitement des événements soit focalisé sur le Bataclan, où l'un de ses journalistes, Guillaume B. Decherf, a trouvé la mort, ainsi que nous en informe un article situé juste avant celui que nous allons analyser.

Je reproduis ci-dessous les premiers paragraphes de l'article du Monde, qui feront l'objet d'un commentaire détaillé :

« Une attaque "complexe" inédite sur le sol français

Les assauts simultanés de commandos-suicides sont typiques des pays où sévissent des guerres asymétriques, Afghanistan, Irak ou Syrie.

C'est une première en France en matière d'attentat. Plusieurs kamikazes se sont fait exploser, dans la soirée du vendredi 13 novembre, au terme d'assauts meurtriers menés aux abords du Stade de France, à Saint-Denis, et dans cinq lieux à Paris, rue de la Fontaine-au-Roi, rue Bichat, boulevard Voltaire, rue de Charonne et dans la salle de spectacle du Bataclan, après la prise en otage du public. Ces attaques, dites "complexes", au regard de modus operandi en plusieurs étapes, sont inspirées d'une forme de violence ayant cours depuis plusieurs années dans des zones de conflit 
telles que l'Afghanistan, l'Irak ou la Syrie, où règne une forme de violence dont la France se croyait jusqu'alors protégée.

L'attaque "complexe" type, telle qu'elle est mise en application en Asie du Sud ou au ProcheOrient par des groupes insurgés, cumule plusieurs actions afin de dérouter les futures victimes en associant très souvent le commando suicide et l'assaut arme à la main. Lors de montages plus ambitieux, elle peut combiner plusieurs attaques simultanées contre un même lieu ou contre des endroits distincts. Les cibles sont minutieusement choisies pour le symbole qu'elles incarnent, mais les personnes qui s'y trouvent sont souvent tuées au hasard et pour leur seule présence dans ces lieux. Le but est de faire le maximum de victimes et de frapper les esprits par la peur.

La police scientifique a rapidement pu identifier les corps des kamikazes coupés en deux au niveau des ceintures d'explosifs.

Selon les premiers éléments de l'enquête de flagrance conduite par le parquet, notamment sur les quatre assaillants qui ont pénétré dans les locaux du Bataclan, ces derniers portaient des ceintures d'explosifs en entrant dans l'établissement. Ils ne les ont déclenchées que lors de l'assaut des forces de l'ordre. Seul un des quatre assaillants ne parviendra pas à se faire sauter. Il a été tué par les hommes de la brigade de recherche et d'intervention lors de l'assaut.

Auparavant, ces quatre hommes se sont appliqués à tuer leurs victimes par balles au moyen d'armes automatiques, en tirant soit sur les gens à terre, soit sur ceux qui tentaient de fuir." "Leur intention était clairement de mourir en martyr", analyse un enquêteur mobilisé tout au long de la nuit sur les lieux du drame ».

On peut définir la structure de cet extrait de la manière suivante : la première partie de l'article (titre, lead, et I re phrase) voit la désignation générique de l'événement; la deuxième partie ( $2^{\mathrm{e}}$ phrase) reprend la mention de l'événement en une seule phrase ; la troisième partie (jusqu'à la fin du $2^{e}$ paragraphe) comprend la définition puis l'explicitation ${ }^{17}$ de la catégorie événementielle générique « attaque complexe »; enfin, la quatrième partie (les trois derniers paragraphes) constitue le compte rendu en tant que tel.

Le récit se veut strictement objectif, il n'y a donc aucune trace énonciative ou de modalisations qui pourraient renvoyer à la subjectivité du journaliste. La modalité du traitement de l'événement apparait dès le titre. Le Monde choisit de désigner les attentats par un terme générique, repris deux fois dans le corps du texte : il s'agit d'une attaque « complexe », qui est certes définie comme « inédite sur le sol français », mais qui est rattachée à des événements «typiques des pays où sévissent des guerres asymétriques ». Nous assistons ainsi à l'avènement d'une nouvelle catégorie événementielle, qui est présentée comme l'information centrale associée à ces attaques. En revanche, aucun des énoncés qui se rattachent aux faits de la nuit précédente ne vise à recréer une scène immersive. On notera d'abord qu'aucun sujet n'est défini par son nom propre ou par des traits idiosyncrasiques. Les seuls sujets singuliers renvoient à une source non spécifiée (un « enquêteur ») et à l'un des assaillants qui « ne parviendra pas à se faire sauter ». Les protagonistes

17 On peut dire que cette partie est dominée par une séquence explicative plutôt que narrative, selon la typologie de Jean-Michel Adam (1992: 127-144). 
sont donc généralement décrits de manière collective, à travers des lexèmes qui renvoient à leur fonction plutôt qu'à leur identité singulière :

- agresseurs : « plusieurs kamikazes », le « commando suicide », les « quatre assaillants 》, « ces quatre hommes » ;

- victimes : les « cibles », le « public », les « victimes », les « gens à terre »;

- protecteurs : la « police scientifique », le « parquet », les « forces de l'ordre », les « hommes de la brigade de recherche et d'intervention ».

Sur le plan de la construction d'un cadre spatio-temporel, on constate que la focalisation sur un lieu singulier, en l'occurrence le Bataclan, n'est pas immédiate, puisque cet endroit est d'abord mentionné en queue d'une liste passant en revue tous les emplacements où se sont déroulés des « assauts meurtriers 》 : aux abords du Stade de France, à Saint-Denis, à la rue de la Fontaine-au-Roi, à la rue Bichat, au boulevard Voltaire et à la rue de Charonne.

Dans le compte rendu, le temps des verbes qui se rattachent aux événements de la veille sont majoritairement énoncés au passé composé (《 se sont fait exploser 》, 《 a pu identifier 》, 《 ont pénétré », 《 ont déclenchées 》, 《 a été tué 》, 《 se sont appliqués »), parfois associés à des imparfaits d'arrière-plan ou marquant la simultanéité du procès (« ces derniers portaient des ceintures d'explosifs», « ceux qui tentaient de fuir »). Ce choix dans les tiroirs verbaux insiste surtout sur le caractère accompli des actions, plutôt que sur leur développement linéaire. On constate d'ailleurs que la structure de l'article ne suit pas la chronologie des événements, même dans les paragraphes focalisés sur l'attaque du Bataclan. Le premier paragraphe désigne globalement les contours de l'événement par le terme générique « assauts meurtriers », mais il est rapidement caractérisé par l'un des aspects les plus frappants de ces assauts: (4) des « kamikazes se sont fait exploser ». En l'absence de connecteurs, la partie proprement narrative, qui commence dans le quatrième paragraphe, adopte un style parataxique ${ }^{18}$ : il débute par (6) l'identification des kamikazes « coupés en deux », avant de remonter le temps pour établir une chronologie des faits qui se sont déroulés dans un lieu particulier: (2) des assaillants pénètrent dans le Bataclan ; (4) ils déclenchent leurs explosifs lors de l'assaut; (5) un terroriste qui n'est pas parvenu à se faire sauter est abattu par les forces de l'ordre. Le paragraphe suivant ajoute deux éléments supplémentaires, qui viennent complexifier cette chronologie : (3) les terroristes ont abattu des victimes avant de se faire sauter ; ( I) leur intention était de mourir en martyr.

Si l'on définit l'ordre des énoncés se rapportant à des contenus narratifs par des lettres, et l'ordre des événements auxquels ils se réfèrent par des chiffres - suivant

${ }^{18}$ Aucune action n'est située précisément dans le temps et l'on constate l'absence d'adverbes soulignant le caractère disruptif des procès (soudain, etc.) ou insistant sur leur enchaînement chronologique ou causal (alors, ensuite, etc.). 
l'usage proposé par Gérard Genette (2007 :27) dans Discours du récit - cela donne la structure suivante :

$$
A 4-B 6-C 2-D 4-E 5-F 3-G 1
$$

Alan Bell (1995: 312, ma traduction) explique ce principe qui consiste, dans l'écriture d'une nouvelle, « à ne pas donner la priorité à l'action ou au processus, mais au résultat », en le rattachant à la logique du flux d'actualité dans lequel s'insère le discours journalistique : « C'est ce principe qui permet aux nouvelles d'être actualisées jour après jour ou heure après heure. S'il y a un nouveau résultat, l'action précédente peut redescendre dans l'histoire ${ }^{19} \gg$. Les manuels d'écriture journalistique préconisent également cet ordre anti-chronologique, qui s'explique aussi par les contraintes d'une lecture hâtive, qui suit rarement le plan linéaire de l'article et élude souvent la fin du texte. La nouvelle apparaît alors comme une sorte de narration qui ne suit pas « la progression vers un point culminant et un dénouement. Habituellement, la nouvelle commence au contraire par... le dénouement. Elle nous apprend tout de suite comment ça s'est terminé. [...] Elle dit l'essentiel d'abord, éliminant le suspense » (Ross, I 990 : 80).

Si le souci de nouer une intrigue ou d'immerger le lecteur dans une histoire est étranger au journaliste du Monde, ce dernier déploie en revanche d'importants efforts pour rendre cet événement plus compréhensible. Ainsi que je l'ai déjà signalé, cela passe, sur un plan axiologique, par le rattachement des protagonistes du drame à des catégories génériques, qui renvoient aux figures des agresseurs, des victimes et des protecteurs. Par ailleurs, dès l'incipit, le journaliste opère un recadrage des événements, qui apparaissaient d'abord comme un drame national « inédit », avant d'être réinterprétés sur une échelle internationale permettant d'identifier une redondance : ces attaques complexes seraient « inspirées d'une forme de violence ayant cours depuis plusieurs années dans des zones de conflit telles que l'Afghanistan, I'Irak ou la Syrie »). Le deuxième paragraphe développe ce parallèle, qui permet non seulement de comprendre la nature de l'agression, mais également son objectif : il est précisé que les « cibles sont minutieusement choisies pour le symbole qu'elles incarnent » et que le « but est de faire le maximum de victimes et de frapper les esprits par la peur ». Cet article apparait donc comme un cas exemplaire du récit informatif : l'immersion et la mise en intrigue sont neutralisées au profit d'une représentation objectivante et généralisante, visant à rendre compréhensible un événement ayant introduit une forte discordance dans le flux de l'actualité.

Le récit proposé par Les Inrockuptibles apparait radicalement différent, aussi bien du point de vue fonctionnel que stylistique. Le titre choisi se situe d'emblée dans le registre des émotions et définit les contours d'un événement singulier : «Trois

\footnotetext{
19 «It is a common principle of news writing that it is not the activity or the process which takes priority but the outcome. It is this principle which enables news stories to be updated day after day or hour by hour. If there is a new outcome to lead with, the previous action can drop down in the story ».
} 
heures d'horreur au Bataclan ». Le chapeau revient sur ce cadre spatio-temporel pour préciser la durée, le lieu et la nature de l'action qui s'y déroule : « De 2 l h40 à 0 h30, la salle de spectacle parisienne a été le théâtre d'un massacre méthodique doublé d'une prise d'otage ». II s'agit aussi de spécifier la source du récit et de légitimer son autorité : « Notre journaliste Marie-Lys Lubrano était au plus près des forces d'intervention ». La signature est donc d'emblée intégrée à la diégèse et le régime énonciatif sera celui d'un récit partiellement homodiégétique, qui se concrétise notamment par l'usage récurrent du pronom « on », qui prend ici une valeur plus inclusive qu'impersonnelle.

Dans ce cas, les contextes plus larges renvoyant aux autres attaques parisiennes et à la problématique générale du terrorisme sont complètement évacués au profit d'une focalisation sur un chronotope ${ }^{20}$ clairement délimité. L'ancrage dans une expérience subjective est marqué non seulement par l'identification de la narratrice, qui est présentée comme un témoin des événements, mais également par le témoignage de différentes victimes (notammentYves, Alice et Julien), qui sont désignées dans le fil du texte par leur prénom et dont les récits respectifs vont permettre de suivre trois fils narratifs, trois drames personnels qui s'insèrent dans la chronologie des attentats. Au total, pas moins de huit noms propres sont mentionnés dans le texte, toujours en lien avec les « victimes » ou les «protecteurs », mais jamais avec les « agresseurs ». Les deux premiers paragraphes sont marqués par l'usage généralisé du présent narratif, qui - par effet d'hypotypose ${ }^{21}$ - peut être considéré comme l'indice le plus élémentaire du processus de déplacement déictique, auquel on peut ajouter le recours fréquent au discours rapporté en style direct, comme si les paroles étaient saisies dans l'actualité de leur énonciation :

« Paris, vendredi, 22 heures. Voilà une demi-heure que la fusillade au Carillon, près du canal SaintMartin, a ouvert le bal des attentats, quand une quinzaine de voitures de police s'engouffrent dans le boulevard Voltaire. L'entrée en est rapidement fermée tandis qu'une flotte d'ambulances de la Croix-Rouge prend position. Avenue de la République, les cafés baissent leurs rideaux métalliques. Flanquées de camions de pompiers garés en travers, les rues du quartier sont méconnaissables. Une sur deux est barrée ; difficile de savoir où se diriger.Très vite, on est cernés par les rubans rouges et blancs, incapables de dire si l'on s'approche ou si l'on s'éloigne de la zone dangereuse.

Soudain, au coin de la rue Crussol et du boulevard Voltaire, un hurlement nous glace :"N'avancez pas !' Le ton sue la panique. Impossible de savoir d'où vient la voix, jusqu'à ce qu'un homme caché derrière une moto bondisse dans la pénombre, arme au poing. On met un peu de temps à percuter qu'il s'agit d'un policier. L'homme menace : "Recule ou je te défonce !" On obéit. II retourne s'accroupir entre les motos, sur le trottoir. Nous sommes devant le 4l, boulevard Voltaire.

${ }^{20}$ Sur l'usage de ce concept bakhtinien dans le domaine de l'analyse du discours journalistique, je renvoie à la thèse défendue par Gilles Merminod (2018a). Je le remercie au passage pour sa relecture du présent article.

${ }^{21}$ L'hypotypose est une « figure de style consistant en une description réaliste, animée et frappante de la scène dont on veut donner une représentation imagée et comme vécue au moment de son expression » (https://fr.wikipedia.org/wiki/Hypotypose). On considère généralement que le recours dans un récit au présent parfois appelé « historique » relève d'un procédé qui peut être rattaché à cette figure rhétorique. 
Le Bataclan est au 50. Les bribes d'infos qui rebondissaient dans les rues étaient vraies : il est 22h40 et une prise d'otages est en cours dans la mythique salle de concerts »..

Ces premières phrases situent la narratrice au cœur de l'action. On relèvera, au début du premier paragraphe, l'association d'une phrase averbale avec une phrase à présentatif, cette association visant probablement à produire un sentiment d'immédiateté. On remarquera ensuite un grand nombre de constructions qui viennent préciser les circonstances des événements rapportés. Le discours est émaillé par plusieurs énoncés descriptifs, qui permettent de se construire une représentation visuelle de la scène («Flanquées de camions de pompiers garés en travers, les rues du quartier sont méconnaissables »), ainsi que par différentes expressions métaphorisantes (« le bal des attentats », « une flotte d'ambulances », « les bribes d'infos qui rebondissaient dans les rues étaient vraies »), qui nous placent dans un registre littéraire. La visualisation de la scène est par ailleurs étayée par une grande photographie occupant la majorité de l'espace couvert par la double page : l'image prise en plan large et en plongée montre une rue où se trouve un groupe de policiers. On peut la rattacher, grâce à une légende, au cadre de l'action, tel qu'il est décrit dans les premiers paragraphes : « Une file de policiers équipés de boucliers aux abords du Bataclan, le 13 novembre $»$. On notera que l'usage du plan large, s'il semble se dissocier du point de vue de la narratrice, apparaît comme un procédé cinématographique typique pour introduire une scène. Deux autres photographies au cadrage plus serré nous placent ensuite à hauteur d'homme, au cœur de l'action : la première montre un homme armé accompagnant deux personnes portant un blessé, et la seconde une série de civils courant dans la rue. La légende nous permet de rattacher ces images aux événements racontés, sans nous permettre de les situer précisément dans le déroulement des faits : « La police tente d'évacuer quelques blessés pendant la prise d'otage ».

Ainsi que nous l'avons déjà suggéré, l'usage répété du pronom « on » dans l'incipit ( « on est cernés par les rubans rouges et blancs, incapables de dire si l'on s'approche ou si l'on s'éloigne de la zone dangereuse ») permet d'inscrire la narratrice dans la communauté indistincte de ceux qui se trouvaient sur place (communauté que le lecteur en régime immersif peut d'ailleurs rejoindre sur un plan imaginaire), et certains témoignages se mêleront d'ailleurs à son discours sans que le marquage de la polyphonie ne soit toujours explicite. Plusieurs subjectivèmes ancrent également la représentation dans le ressenti du « je narré » ou de la foule présente sur place ( « un hurlement nous glace », « le ton sue la panique »), par ailleurs, des adverbes tels que « voilà », « soudain », ou « très vite » dynamisent la durée et l'inscrivent dans l'actualité des événements. Le récit vise donc la reconstitution d'une scène focalisée sur l'expérience subjective de plusieurs témoins du drame, plutôt que d'accroître notre compréhension objective des faits en s'appuyant sur le point de vue rétrospectif de la journaliste qui rédige ce récit cinq jours plus tard. Au lieu de chercher à remédier aux limitations du savoir des personnages plongés au cœur des événements, la narration exploite ces lacunes pour dynamiser le récit : par exemple, il faut attendre la fin du deuxième paragraphe pour que le « je narré » se voie confirmer la nature de l'événement et sa localisation, alors que le « je 
narrant » ne peut évidemment pas ignorer de telles informations. De manière semblable, l'explicitation de l'identité ou du rôle actantiel de certains personnages est stratégiquement retardée pour produire un effet de curiosité relativement bref mais particulièrement efficace dans le passage suivant : « Impossible de savoir d'où vient la voix, jusqu'à ce qu'un homme caché derrière une moto bondisse dans la pénombre, arme au poing. On met un peu de temps à percuter qu'il s'agit d'un policier $\gg$.

Suite à cette introduction, dont la durée est précisément délimitée entre 22h00 et 22h40, on observe ensuite une analepse dramatisée ${ }^{22}$, qui nous fait basculer dans un autre point de vue : celui des témoins qui ont vécu le drame en direct, à l'intérieur du Bataclan : « Les bribes d'info qui rebondissaient dans les rues étaient vraies : il est $22 \mathrm{~h} 40$ et une prise d'otages est en cours dans la mythique salle de concert. À l'intérieur, tout le monde l'a compris depuis une heure ». À partir de ce moment, le récit se focalise sur trois témoins principaux, désignés par leur prénom et caractérisés par des traits idiosyncrasiques qui les distinguent de la foule : il y a d'abord Yves, un auteur de 36 ans, qui accompagne Elea, une amie ; ensuite Alice, 23 ans, dont le récit est relayé par sa sœur Cécile car elle est trop éprouvée pour témoigner directement ; enfin Julien, qui est défini comme « grand, baraqué, le crâne rasé » et qui « avait emmené son neveu au concert, avec deux copains », Xavier et Mathieu. II faut préciser que les témoignages sont parfois rapportés en style direct, mais ils sont le plus souvent transformés en récit narré au présent, dans une forme qui se confond avec l'écriture de la journaliste.

À partir du flashback, le déroulement respecte strictement la chronologie des événements, qui est très précisément reconstruite dans le fil du récit, probablement en s'appuyant sur l'heure inscrite dans la mémoire des téléphones portables utilisés par les témoins. L'agencement temporel de de l'article peut ainsi être reconstruit de la manière suivante :

22h00 à 22h40 Exposition in medias res (focalisation sur l'espace extérieur au Bataclan)

2 Ih00 à 22h40 Analepse dramatisée (focalisation sur l'espace intérieur au Bataclan)

2 I h00 Yves se place près de la scène

2 Ih40 Attaque des terroristes

22h08 Yves envoie un SMS à sa petite amie pour la rassurer

22h20 Yves se réfugie sur le toit et appelle ses parents

22 Dans le cas présent, l'analepse s'intègre dans la dynamique du récit en succédant à une introduction énigmatique engendrant de la curiosité, ce qui motive le retour en arrière. La scène rétrospective joue ainsi le rôle d'une « analepse complétive » dans la terminologie de G. Genette (2007 : 4I). Sur la distinction entre « analepse dramatisée » (ou flashback) et « analepse allusive » (sans immersion), voir Baroni (2016b). 
22h40 à 0Ih45 Retour au « présent » de la journaliste (focalisation sur l'espace extérieur au Bataclan)

23h00 Départ du groupe d'intervention

23h 13 Arrivée d'un fourgon de la BRI

23h 16 Des policiers entrent dans le Bataclan

23h 19 Alice est sauvée et appelle sa sœur

23h40 Débriefing avec un négociateur de la BRI

23h45 Établissement d'une liste de contacts des victimes piégées à l'intérieur

00h00 Assaut final

00h20 Série de coups de feu et d'explosions

OIhI5 Yves et Xavier sortent du Bataclan

O Ih45 Julien apprend que son neveu et Mathieu ont survécu

Ce qui frappe dans cette structure, ce n'est pas seulement le respect de la chronologie, mais aussi la manière dont le récit suit précisément le déroulement de plusieurs destins entrecroisés, en exploitant pleinement les incertitudes éprouvées par les personnages plongés au cœur du drame, que ce soit à travers leur interprétation erronée des événements (« comme les autres, aux premiers coups de feu, elle a pensé à des pétards. Jusqu'à ce qu'elle voie le groupe se barrer de la scène en courant ») ou par la manière dont ils envisagent des virtualités qui ne se réaliseront pas :

« Dans la fosse, Alice remarque qu'il y a un petit espace sous la cabine des ingés son ; peut-être qu'elle pourrait s'y glisser. À côté, les assaillants chuchotent en français. Elle entend "grenades" et comprend qu'ils veulent faire sauter la salle. Se fourrer dans un trou n'est pas forcément une bonne idée s'il y a une bombe, ou s'il faut évacuer. Elle ne bouge pas ».

La tension narrative qui accompagne le récit de Julien est particulièrement frappante, dans la mesure où la narratrice choisit de ne dénouer le suspense que dans la dernière phrase de son article. On découvre Julien au moment où les premiers rescapés du Bataclan sont évacués de la salle, alors qu'il téléphone à sa sœur pour essayer de la rassurer :

« Julien, lui, essaie de rassurer sa sœur au téléphone. "Mais je ne l'ai pas laissé là-bas ! j’ai été littéralement emporté par un mouvement de foule vers la sortie de secours ! Je ne maîtrisais rien !" La gorge écrasée par l'angoisse, il ne sait plus comment expliquer à sa sœur qu'il n'a pas abandonné son neveu. Dans les minutes qui ont suivi l'attaque, il a réussi à le joindre et lui a demandé de se cacher et d'éteindre son portable, pour ne pas se faire repérer. Quelques nouvelles lui parviennent par SMS : un de ses amis, Mathieu, s'est caché sous les toits avec son neveu ».

Pour son malheur, Julien ne sera informé du sort de son neveu qu'une demi-heure après la fin des opérations de sauvetage. Comme la narratrice choisit de repousser 
cette révélation dans le dernier paragraphe de son article, elle offre à son récit un dénouement sous forme de happy end cathartique :

« Julien lui, ne peut plus appeler sa sœur. II n'a pratiquement plus de batterie et absolument plus de mots. Le Bataclan est évacué depuis une demi-heure, Xavier l'a appelé tout de suite. Mais pas Mathieu ou son neveu. Julien vacille. Les journalistes complètent le tableau : il y a eu sept attentats dans Paris et on parle de près d'une centaine de morts rien qu'au Bataclan. Julien n'entend pas. Enfin son téléphone sonne. II décroche et se met à pleurer. Son neveu est sorti avec Mathieu, ils vont bien ; ils ont réussi à rester planqués sous le toit. "Putain, il est fort ce petit mec quand même", sourit Julien à travers ses larmes ».

Si la valeur informative de l'article du Monde ne pose guère problème, on pourrait en revanche ressentir un certain malaise face au récit immersif publié dans Les Inrockuptibles, qui rappelle les effets racoleurs de la presse à sensation. Marie Vanoost (2016: \$9) a montré cependant que le journalisme narratif n'est pas dénué de préoccupations éthiques, ainsi que l'affirment de nombreux journalistes qui pratiquent ce genre :

«L'information, hors de tout contexte humain, constitue une abstraction que l'on peut facilement ignorer ou ne pas pleinement comprendre. Les journalistes narratifs sont donc convaincus d'offrir au lecteur une compréhension plus large et plus profonde du monde qui nous entoure, d'atteindre un niveau de configuration plus élevé que le journalisme "classique" ».

Là où l'article du Monde visait à colmater une brèche introduite par un événement disruptif, en inscrivant la discordance du drame dans un cadre plus concordant, qui permet de reconnaître des phénomènes plus généraux, le récit des Inrockuptibles revient au contraire sur les circonstances de l'attaque du Bataclan, non pour les éclairer, mais pour rendre justice à l'expérience vécue par les victimes. Au lieu d'élargir l'échelle pour transcender la singularité de l'événement, il s'agit cette fois de nous replacer au plus près du vécu des protagonistes et des témoins pour en faire éprouver l'impact émotionnel. Le choix d'orienter le récit selon le point de vue des victimes, et non selon celui des agresseurs ou des protecteurs, prend alors une importance cruciale sur un plan éthique. Le savoir mis en jeu est fondé sur une compréhension plus profonde, qui se situe au niveau de l'empathie plutôt qu'au niveau d'une connaissance distanciée, qui rendrait les événements racontés plus facilement interprétables. La narration immersive rejoint alors les propos d'Áron Kibédi Varga (1989: 73) quand il affirme que le savoir que le récit est appelé à transmettre « n'est pas un savoir scientifique au sens strict et positiviste de ce terme, mais plutôt un savoir humain, une accumulation et un prolongement des expériences du lecteur ».

Outre les métissages qui peuvent exister entre ces deux formes contrastées dont je viens d'esquisser les caractéristiques fonctionnelles et formelles, je n'ai aucune prétention à avoir épuisé les possibles narratifs qui s'offrent aux journalistes quand il s'agit de traiter un événement dramatique. II s'agissait néanmoins d'offrir un modèle heuristique permettant de faire évoluer le cadre interprétatif dérivé des travaux de Paul Ricœur. Je voulais montrer comment une typologie plus fine permettait de mettre en évidence certains phénomènes de surface que l'on peut 
rattacher alternativement à un style informatif ou, au contraire, à un style immersif, tout en rattachant ces formes narratives aux fonctions qu'elles remplissent dans leur contexte médiatique respectif.

Mais rien n'oblige à limiter notre horizon à ces deux pôles : il est notamment possible d'esquisser une troisième catégorie médiatique, que l'on pourrait désigner comme celle des récits immergés dans l'actualité, c'est-à-dire ceux qui s'insèrent à la fois dans le flux d'un événement inachevé ou énigmatique, et dans une série d'épisodes orientés vers un dénouement possible, ce qui permet de structurer une intrigue qui se déploie à l'échelle transtextuelle d'un feuilleton médiatique (Baroni, 20।6c : § I7). Dans de tels cas, la question se pose moins dans les termes d'une alternative entre style immersif ou style informatif, que dans ceux d'un jeu de contraintes posées à l'écriture par la situation du narrateur, dont la perspective est effectivement limitée, ce qui l'oblige à attendre des développements ultérieurs pour raconter le dénouement. Dès lors, la distinction entre temporalité intra- et extra-diégétique n'a plus la même pertinence : l'ancrage du discours est également un ancrage dans un événement qui se raconte au jour le jour.

On constate ainsi que le soir même des attentats, le site du Monde a publié un article proposant de « suivre en direct » le déroulement de la situation. Dans ce premier compte rendu, d'abord mis en ligne le 13 novembre à $22 \mathrm{h0} 4$ (soit environ 40 minutes après le début des attentats), puis mis à jour jusqu'au 15 novembre à 22h3l, le récit n'est pas seulement focalisé sur le passé. Certes, une carte permet de situer géographiquement et chronologiquement le déroulement des attentats, qui sont littéralement saisis sous la forme d'une configuration visuelle, mais on trouve aussi un point sur la situation actuelle - le « bilan provisoire » est énoncé au présent, car il peut encore évoluer - et l'évocation de conséquences futures, qui sont articulées sous la forme prospective du conditionnel :

« Un bilan provisoire fait état d'au moins 129 morts et 352 blessés, dont beaucoup dans un état grave, selon le procureur de la République de Paris, François Molins, mais il pourrait s'alourdir. Sept terroristes sont morts, tous après avoir actionné leurs ceintures explosives.

L'organisation État islamique a revendiqué ces meurtres, samedi. François Hollande a promis que "la France sera[it] impitoyable à l'égard des barbares". II a décrété un deuil national de trois jours et a annoncé que « tous les dispositifs » de sécurité seraient renforcés à leur niveau maximal.

Suivez la situation et les réactions en direct sur LeMonde.fr »

Pour le feuilleton médiatique, chaque épisode renvoie à la portion de l'histoire qui vient de se dérouler, mais il faut envisager aussi une histoire inachevée et plus englobante, ce qui implique non seulement d'inclure des rappels d'événements plus anciens, mais aussi de rendre compte des événements actuels (ceux marqués par une forme de suspension ou d'attente) et d'évoquer des développements ultérieurs, qui sont généralement articulés, à ce stade, sous la forme d'hypothèses. Si l'on veut esquisser une définition de ce récit immergé dans l'actualité, il faut dès lors intégrer à l'analyse ces trois aspects : passé, présent et futur, les deux dernières perspectives temporelles ayant été généralement négligées par les 
approches formalistes, dont les concepts ont été forgés à partir de récits dont on présupposait l'unité rétrospective et la complétude. On se situe alors de plain-pied dans ce que Paul Ricœur appellerait la préfiguration ou la mimèsis I, mais celle-ci est saisissable à travers des formes discursives concrètes, et non comme un simple pré-cadrage de l'expérience. Dans notre exemple, on constate que la logique du direct appelle d'autres formats que celui du journalisme traditionnel, qui reste soumis à un rythme de diffusion périodique quotidien ou hebdomadaire. Les fils d'actualité diffusés sur l'internet ou les chaînes d'information en continu prennent alors le relais des médias traditionnels et produisent une expérience narrative de plus en plus marquée par la multimodalité et la coordination transmédiatique.

Face aux inquiétudes engendrées par l'infotainment et les flux d'information en direct ${ }^{23}$, je tiens cependant à préciser qu'il serait réducteur de rattacher le feuilleton médiatique exclusivement à ces nouveaux formats ou de l'associer à un inévitable affaiblissement du travail de configuration, qui conduirait à une perte de sens et à une confusion généralisée. En effet, pour autant que l'événement ne soit pas pris dans une actualité aussi brûlante qu'une série d'attentats, il faut reconnaître l'existence de feuilletons médiatiques qui se déploient sur une très large échelle temporelle, ce qui les rend parfaitement adaptables aux formats périodiques traditionnels et à un journalisme d'investigation. Pour prendre des exemples récents, le suspense politique lié à la négociation de la dette grecque (20 I0-20 I I), au Brexit (20162018) ou au destin des migrants embarqués à bord de l'Aquarius (2016-2018) ont tenu en haleine les lecteurs des journaux pendant des jours, des semaines ou des années entières, ce qui a laissé tout loisir aux journalistes pour rédiger des épisodes hautement informatifs au sein d'une actualité qui n'avait nul besoin d'être dramatisée pour tenir en haleine le public jusqu'à son éventuel dénouement. II y a quelques enseignements à tirer du fait que ces récits immergés, rythmées par des nœuds, des rebondissements et des dénouements plus ou moins imprévisibles, ressemblent beaucoup, sur une échelle globale, aux récits immersifs, alors que sur une échelle locale, chaque épisode peut parfaitement se conformer aux modalités discursives des récits informatifs (voir Baroni, 2016c;2016d).

Je n'ai pas la place dans ces lignes pour entrer dans le détail de l'analyse de ce type de narrativité immergée, que nous avons étudiée au sein du laboratoire d'analyse du récit de presse (Larp) de l'Université de Fribourg en Suisse (Baroni, Pahud, Revaz, 2006 ; Revaz, Baroni, 2007 ; Revaz 2009 ; Baroni, 20 I 6c), mais j'aimerais en revanche insister sur l'importance de faire évoluer la théorie du récit en tenant compte des phénomènes liés à la sérialisation de la production et de la diffusion des histoires.

\footnotetext{
${ }^{23}$ Ainsi que l'affirme Marc Lits $(2010: \S 1)$, « le récit médiatique semble donc menacé par l'information en direct. En outre, comme celle-ci gagne du terrain, tant par le développement des chaînes d'information en continu (en radio comme en télévision) que dans une accélération de la couverture médiatique de l'information rendue possible par les progrès technologiques en transmission d'images et encouragée par la concurrence entre chaînes (la valeur est dans la primeur de l'information plutôt que dans son interprétation), la possibilité d'une correcte mise en œuvre de la deuxième mimèsis doit désormais être rediscutée ».
} 
Cette narrativité sérielle apparaît aujourd'hui de plus en plus centrale dans notre paysage médiatique et elle mobilise des mécanismes qui lui sont propres ${ }^{24}$, ce qui devrait nous amener à reconsidérer certains acquis de la théorie structuraliste et à poursuivre nos efforts de théorisation (Escola, 2010 ; Goudmand, 2013 ; Baroni, Jost, 2016 ; Ryan, 2018).

\section{Vers une troisième génération de narratologues?}

La réflexion qui précède visait à montrer qu'une conception trop étroite de la narrativité, ou trop large de ce que l'on considère comme le processus de mise en intrigue, conduisait à négliger des traits formels et fonctionnels extrêmement importants qui permettent de caractériser des manières différentes de raconter des événements réels ou inventés. Ainsi que l'affirme Gregory Currie (2010), les récits sont des artefacts communicationnels façonnés dans des contextes discursifs qui posent des contraintes spécifiques sur le sens et la forme de l'acte narratif. Je ne peux donc qu'abonder dans le sens de Marie Vanoost (2016: §49) lorsque cette dernière affirme que, « des contraintes, des visées, des effets - réels ou espérés - différents impliquent, assez logiquement, une poétique au moins en partie différente. La narratologie ne peut que s'enrichir au contact de récits qui sortent de son domaine d'analyse privilégié - celui de la fiction ».

La typologie présentée dans ces lignes ne définit que quelques pôles ou modèles prototypiques de la narrativité qui mériteraient d'être affinés en les confrontant à des objets empiriques aussi diversifiés que possibles. Je n'ai fait qu'effleurer la dimension multimodale des formes narratives en évoquant les rapports texteimage dans l'article des Inrockuptibles ou l'infographie du Monde, mais elle mériterait d'être approfondie dans un contexte marqué par une interdépendance de plus en plus forte entre des supports médiatiques différenciés. II me semble néanmoins que l'analyse proposée permet de dégager, au sein des discours journalistiques, des formes narratives représentant le passé selon des modalités contrastées, là où un modèle trop simple aurait plutôt tendance à écraser ces différences.

Pour conclure, j'aimerais revenir sur la question de la place occupée par la théorie du récit au sein des institutions académiques, qui a fait l'objet des principales critiques qui m'ont été adressées dans le cadre de cet échange. La question du lieu d'ancrage institutionnel de la narratologie est, à mes yeux, secondaire. La seule chose qui compte, c'est qu'il soit possible de trouver un endroit favorable pour mener un travail de théorisation des formes narratives, ce qui implique de maintenir, d'un côté, un dialogue avec la famille restreinte des narratologues, tout

${ }^{24}$ Je précise que dans les travaux du Larp, nous avons insisté sur les différences importantes qui existent entre la sérialisation dans le discours journalistique et les fictions sérielles, qui tiennent autant aux contraintes de production qu'aux formes textuelles et à la réception des discours (Baroni, Pahud, Revaz, 2006 ; Baroni, 2016c). 
en continuant, d'un autre côté, à échanger avec des chercheurs qui s'inscrivent dans le champ plus vaste des études narratives. J'ai évoqué un ancrage dans les départements de littérature pour une raison purement historique : c'est dans ce contexte que s'est constituée une tradition de recherche plongeant ses racines dans la poétique et la rhétorique classique, se prolongeant dans le formalisme russe et le structuralisme français, et s'élargissant aujourd'hui pour intégrer une réflexion transgénérique et transmédiale (Thon, 2016; Baroni, 20।7b ; Ryan, 2018).

S'inscrire dans cette tradition me semble être une nécessité si l'on veut éviter de faire bégayer l'histoire ou de réinventer continuellement la roue, mais il ne s'agit nullement d'affirmer que les œuvres littéraires constitueraient des objets plus complexes ou plus intéressants pour la narratologie que les récits que l'on rencontre dans d'autres médias (cinéma, télévision, bande dessinée, roman-photo, jeux vidéo, etc.) ou dans d'autres sphères d'activité (journalisme, publicité, politique, didactique, droit, médecine, etc.). J'estime cependant que le rattachement de la narratologie à un département de littérature permettrait d'identifier un lieu où cette tradition théorique et critique a pris son essor, et pourrait continuer de prospérer en élargissant son spectre, tout en renouvelant l'attractivité d'une discipline en crise. II pourrait être utile, pour sortir de cette crise, de rappeler que les études littéraires ont longtemps constitué le lieu où une réflexion générale sur les formes narratives, sur les fictions et sur l'immersion dans des mondes racontés a pu se développer.

Si les narratologues de la première génération ont été d'abord des littéraires - je pense naturellement à ces deux figures tutélaires qui viennent de nous quitter : Tzvetan Todorov et Gérard Genette, mais aussi à Roland Barthes et à quelques autres -, ceux de la deuxième génération ont essaimé dans des domaines très variés, le spectre s'étendant des sciences du langage aux études culturelles, en passant, entre autres, par le journalisme et les sciences de l'information et de la communication. II y a néanmoins un fil rouge auquel se sont tenus tous ces chercheurs remarquables : l'effort continu pour faire évoluer la théorie du récit, ce qui exige à la fois la capacité à inscrire sa réflexion dans une tradition théorique et critique, tout en nouant un dialogue avec une communauté de chercheurs de plus en plus vaste et diversifiée. La dette commune de ces théoriciens du récit envers les travaux des narratologues de la première génération a longtemps constitué un lien important pour unifier cette famille composite, alors que la diversité de ses membres a en même temps permis de faire des avancées considérables. L'Observatoire des récits médiatiques de l'université catholique de Louvain a été l'un des fers de lance d'une narratologie relevant le défi de la transmédialité, mais cette orientation de recherche est fragile au sein d'une école de journalisme, et si l'on se soucie de la santé de la théorie du récit, alors on peut juger inquiétant le fait que le sigle de l'ORM ait été récemment redéfini comme Observatoire de recherche sur les médias et le journalisme 25 .

$\overline{25}$ Voir la page institutionnelle de l'ORM : http://www.comu.be/orm. Consulté le 20/12/20 I 8. 
Alors que la deuxième génération des narratologues est en train d'arriver à l'âge de la retraite, la question est de savoir comment s'organisera une troisième génération de chercheurs. Ainsi que le préconise Marc Marti (2017), le dialogue interdisciplinaire est certes fondamental, de même que le maintien ou le développement de réseau ${ }^{26}$, de revues ou de collections, mais je pense que l'identification à une discipline de recherche passe aussi par l'existence d'ancrages institutionnels offrant la possibilité de préserver et de faire fructifier un héritage théorique commun. En 2013, la troisième conférence du réseau européen de narratologie (ENN) s'interrogeait sur la manière dont la théorie du récit évolue et sur la question de savoir si cette dernière s'orientait vers une consolidation ou vers une diversification (Hansen et al., 2017). Je pense que si nous suivons le chemin de la diversification extrême et que nous ne parvenons pas à trouver de terrain favorisant une convergence disciplinaire, le risque est grand de devenir incapables de poursuivre un débat d'idées qui seul permet de faire avancer la théorie. La diversification risque alors de devenir un éparpillement ou une dissolution. Peutêtre qu'un paysage académique débarrassé de la narratologie ne serait pas une catastrophe en soi, les perspectives offertes aux études narratives demeurant nombreuses, mais je pense malgré tout qu'un affaiblissement de la théorie du récit ne serait pas une bonne nouvelle dans un contexte marqué par une montée en puissance de ce qu'Yves Citton (2010) a appelé les « mythocraties ».

\section{Références}

Adam J.-M., 20 I I, Genres de récits. Narrativité et généricité des textes, Louvain-la-Neuve, Éd. L'Harmattan-Academia.

Adam J.-M., 1992, Les Textes, types et prototypes. Récit, description, argumentation, explication et dialogue, Paris, Nathan, 1997.

Baetens J., 2017, « Nouvelle narratologie, nouveau récit », Questions de communication, 31, pp. $231-244$.

Baroni R., 2007, La Tension narrative. Suspense, curiosité et surprise, Paris, Éd Le Seuil.

Baroni R., 2009, L'CEuvre du temps. Poétique de la discordance narrative, Paris, Éd Le Seuil.

Baroni R., 20 I0, « Ce que l'intrigue ajoute au temps. Une relecture critique de Temps et récit de Paul Ricœur », Poétique, 163, pp. 361-382.

Baroni R., 2016a, « L'empire de la narratologie contemporaine, ses défis et ses faiblesses », Questions de communication, 30, pp. 219-239. Accès : https://www.cairn.info/revuepoetique-20। 0-3-page-36I.htm.

Baroni R., 2016b, « Dramatized Analepsis and Fadings in Verbal Narratives », Narrative, 24 (3), pp. 31 I-329.

${ }^{26}$ Je profite de cette occasion pour signaler la création du réseau des narratologues francophones (RéNaF), annoncé dans mon article précédent (2016a) : https://wp.unil.ch/narratologie. Consulté le 20/12/2018. 
Baroni R., 2016c, « Un feuilleton médiatique forme-t-il un récit? », Belphégor, I4 (I ). Accès : https://journals.openedition.org/belphegor/660.

Baroni R., 20 I6d, « Le cliffhanger : un révélateur des fonctions du récit mimétique », Cahiers de narratologie, 31. Accès : http://journals.openedition.org/narratologie/7570.

Baroni R., 2017a, Les Rouages de l'intrigue. Les outils de la narratologie postclassique pour l'analyse des textes littéraires, Genève, Slatkine.

Baroni R., 20 I7b, « Pour une narratologie transmédiale », Poétique, I82, pp. I55- 175.

Baroni R., Jost F., 2016, coords, « Repenser le récit avec les séries télévisées », Télévision, 7. Accès : https://uww.cairn.info/revue-television-2016-1.htm.

Baroni R., Pahud S., Revaz F., 2006, « De l'intrigue littéraire à l'intrigue médiatique : le feuilleton Swissmetal », A Contrario, 4 (2), pp. 125- 143.

Bell A., 1995, « News Time », Time \& Society, 4 (3), pp. 305-328.

Carrard P., 2012, «The Distinction of Historiography: Dorrit Cohn and Referential Discourse », Narrative, 20 ( I), pp. |25-131.

Citton Y., 20 I 0, Mythocraties. Storytelling et imaginaire de gauche, Paris, Amsterdam.

Currie G., 20 I0, Narrative and Narrators. A Philosophy of Stories, Oxford, Oxford University Press.

Dannenberg H., 2008, Coincidence and Counterfactuality. Plotting Time and Space in Narrative Fiction, Lincoln, University of Nebraska Press.

Duchan J. F., Bruder G.A., Hewitt L. E., 1995, Deixis in Narrative. A Cognitive Science Perspective, Hillsdale, Lawrence Erlbaum Associates.

Escola M., 20 I0, « Le clou deTchekhov. Retours sur le principe de causalité régressive », Fabula. Accès : http://www.fabula.org/atelier.php?Principe_de_causalite_regressive\#_ftnref2 I.

Fleury B., Walter J., 2017, « La narratologie dans tous ses états », Questions de communication, 31, pp. 183-198.

Fludernik M., 1996, Towards a "Natural" Narratology, Londres, Routledge.

Genette G., 2007, Discours du récit, Paris, Éd. Le Seuil.

Goudmand A., 2013, « Narratologie du récit sériel », Proteus, 6, pp. 8 I-89. Accès : http://www. revue-proteus.com/articles/Proteus06- I 0.pdf.

Hansen P. K. et al., 2017, Emerging Vectors of Narratology, Berlin, De Gruyter.

Jost F., 2017, « À quelles conditions est-il possible de faire une narratologie comparée ? », Questions de communication, 31 , pp. 265-278.

Kibédi Varga Á., 1989, Discours, récit, image, Bruxelles, P. Mardaga.

Kreiswirth M., 1995, «Trusting the Tale:The Narrativist Turn in the Human Sciences », New Literary History, 23 (3), pp. 629-657.

Kukkonen K., 2014, « Bayesian Narrative: Probability, Plot and the Shape of the Fictional World », Anglia, I 32 (4), pp. 720-739.

Lits M., 2008, Du Récit au récit médiatique, Bruxelles, De Boeck.

Lits M., 20 I0, « L'impossible clôture des récits multimédiatiques 》, A Contrario, 13, pp. I 13 124. Accès : https://www.cairn.info/revue-a-contrario-20 I0- I-page- I I 3.htm. 
Marion P., 1997, « Narratologie médiatique et médiagénie des récits », Recherches en communication, 7, pp. 61 -88.

Marti M., 2017, « De la narratologie littéraire à la narratologie générale : l'empire et ses limes », Questions de communication, 31, pp. 199-214.

Merminod G., 2018a, La Trajectoire d'une histoire. Formes, usages et idéologies du raconter journalistique, thèse en lettres, Université de Lausanne.

Merminod G., 2018b, «Telling Stories From the Newsroom: A Linguistic Ethnographic Account of Dramatization in Broadcast News », Cahiers de l'ILSL, 54, pp. 25-54.

Patron S., 2018, Introduction à la narratologie postclassique. Les nouvelles directions de la recherche sur le récit,Villeneuve d'Ascq, Presses universitaires du Septentrion.

Pedot R., 2008, « Intriguer, au-delà de l'intrigue », Tropismes, I 5, pp. 23-48.

Rabatel A., 2017, «Des récits en général, de la narratologie en particulier 》, Questions de communication, 31 , pp. 245-264.

Revaz F., 2009, Introduction à la narratologie. Action et narration, Bruxelles, De Boeck/Duculot.

Revaz F., Baroni R., 2007, « Le fait divers sérialisé, un feuilleton médiatique », Les Cahiers du journalisme, 17, pp. 194-209.

Ricœur P., 1983-1985, Temps et récit, 3 tomes, Paris, Éd. Le Seuil.

Ricœur P., 1990, Soi-même comme un autre, Paris, Éd. Le Seuil.

Ross L., 1990, L'Écriture de presse. L'art d'informer, Boucherville, G. Morin.

Ryan M.-L., 200I, Narrative as Virtual Reality. Immersion and Interactivity in Literature and Electronic Media, Baltimore, Johns Hopkins University Press.

Ryan M.-L., 20 I 8, « Sur les fondements théoriques de la narratologie transmédiale », pp. I47166, in : Patron S., dir., Introduction à la narratologie postclassique,Villeneuve d'Ascq, Presses universitaires du Septentrion.

Saurier D., Vallée O., 2017, "Récits, figures et chemins de traverse », Questions de communication, 31, pp. 279-290.

SchmittA., 201 7, « La pratique narratologique », Questions de communication, 31, pp. 21 5-229.

Thon J.-N., 201 6, Transmedial Narratology and Contemporary Media Culture, Lincoln, University of Nebraska Press.

Vanoost M., 2016, « Journalisme narratif : des enjeux contextuels à la poétique du récit », Cahiers de narratologie, 31 . Accès : https://journals.openedition.org/narratologie/7543.

Wagner F., 2017, « Marbot à l'épreuve de la relecture », Poétique, I82, pp. 259-278.

White H., 20 I7, L'Histoire s'écrit. Essais, recensions, interviews, trad. de l'anglais par P. Carrard, Paris, Éd. de la Sorbonne. 


\section{Sources}

Anonyme, 2015, «Attaques à Paris : le point sur l'enquête et le déroulé des attaques », Le Monde.fr, 13 nov. à 22h04, mis à jour le 15 nov. à 22h31. Accès : https://www.lemonde.frl societe/article/20 I 5/ I I/I3/fusillade-meurtriere-a-paris_4809485_3224.html. Consulté le 24/05/2018.

Follorou J., 2015, « Une attaque "complexe" inédite sur le sol français », Le Monde, 14 nov. Accès : https://www.lemonde.fr/attaques-a-paris/article/20 I5/I I//4/un-attentatcomplexe-inedit-sur-le-sol-francais_4809754_4809495.html. Consulté le 24/05/20 I8.

Lubrano M.-L., 2015, «Trois heures d'horreur au Bataclan », Les Inrockuptibles, I8-24 nov., pp. $10-14$. 